\title{
Hardy spaces of exact forms on domains
}

\author{
ZENGJIAN LOU
}

We consider Hardy spaces $\mathcal{H}_{z, d}^{1}\left(\Omega, \wedge^{k}\right)$ of exact $k$-forms supported in strongly Lipschitz domains $\Omega$ of $N$-dimensional Euclidean space $\mathbb{R}^{N}$ and aim to give their atomic decompositions, characterize their dual spaces, and establish "div-curl" type theorems on domains $\Omega$.

When $k=2$ and $\Omega=\mathbb{R}^{3}, \mathcal{H}_{z, d}^{1}\left(\Omega, \wedge^{k}\right)$ reduces to a divergence-free Hardy space on $\mathbb{R}^{3}$. We exhibit a divergence-free atomic decomposition of the space and give a "div-curl" type theorem on $\mathbb{R}^{3}$. We also investigate some applications of the "div-curl" type theorem to coercivity properties of some polyconvex quadratic forms which come originally from the linearization of polyconvex variational integrals studied in nonlinear elasticity on $\mathbb{R}^{3}$. When $\Omega$ is the whole space $\mathbb{R}^{N}$, the upper half-space $\mathbb{R}_{+}^{N}$, a special Lipschitz domain or a bounded strongly Lipschitz domain in $\mathbb{R}^{N}$, we prove atomic decompositions of $\mathcal{H}_{z, d}^{1}\left(\Omega, \wedge^{k}\right)$ as sums of exact atoms with supports in $\Omega$. These results follow from tent space arguments along with a reproducing identity. We then use those decompositions to characterize dual spaces of $\mathcal{H}_{z, d}^{1}\left(\Omega, \wedge^{k}\right)$. In addition we establish "div-curl" type theorems on $\Omega$ with applications to coercivity.

The content of the thesis is roughly as follows. The first chapter contains preliminary material on Hardy spaces, $B M O$ spaces, tent spaces and Sobolev spaces. The only original feature of the chapter seem to be an observation that the curl operator is surjective from the Sobolev space $H_{0}^{1}\left(\Omega, \mathbb{R}^{3}\right)$ to a subspace of $L^{2}\left(\Omega, \mathbb{R}^{3}\right)$ when $\Omega$ is a smooth and simply-connected domain in $\mathbb{R}^{3}$.

The second chapter deals with estimates of Jacobian determinants on a bounded strongly Lipschitz domain $\Omega$ in $\mathbb{R}^{2}$. The corresponding estimate on $\mathbb{R}^{2}$ is due to Coifman, Lions, Meyer and Semmes [1]. Also in this chapter, we give a decomposition theorem of $\mathcal{H}_{z}^{1}(\Omega)$ into "Jacobian" quantities. A similar decomposition of $\mathcal{H}^{1}\left(\mathbb{R}^{N}\right)$ into "div-curl" quantities was obtained in [1, Theorem III.2].

In Chapter III, we consider the three-dimensional divergence-free Hardy space. We prove a divergence-free atomic decomposition of the space: any function in the space

Received 17th April, 2003

Thesis submitted to the Australian National University, June 2002. Degree approved, Decemember 2002. Supervisor: Professor Alan $\mathbf{M}^{\mathbf{c}}$ Intosh.

Copyright Clearance Centre, Inc. Serial-fee code: 0004-9727/03 $\$ A 2.00+0.00$. 
can be decomposed into a sum of divergence-free atoms. Using the decomposition we characterize its dual: the $B M O$-type space. Applying the duality relationship between the divergence-free Hardy space and the $B M O$-type space we establish a "div-curl" type theorem, which is used to prove some coercivity properties of certain polyconvex quadratic forms. The divergence-free Hardy space on $\mathbb{R}^{N}$ was studied by Gilbert, Hogan and Lakey in [2]. They gave its atomic decomposition by using a divergence-free wavelet decomposition of the divergence-free $L^{2}\left(\mathbb{R}^{N}, \mathbb{R}^{N}\right)$ space [3]. The idea we used here is different from that in [2] and is valid for cases of domains and forms. Our proof relays on a reproducing identity and techniques in harmonic analysis, including atomic decompositions of tent spaces, Whitney decompositions and reflection maps.

The atomic decomposition and the "div-curl" type theorem in Chapter III are generalized to Hardy spaces $\mathcal{H}_{d}^{1}\left(\mathbb{R}^{N}, \wedge^{k}\right)$ in Chapter IV. In this chapter, we also prove a decomposition theorem of $\mathcal{H}_{d}^{1}\left(\mathbb{R}^{N}, \wedge^{k}\right)$ into " $d u \wedge d v$ " quantities, which is an extension of [1, Theorem III.2].

Chapter $\mathrm{V}$ is perhaps the heart of the thesis. In this chapter, we first consider a divergence-free Hardy space on the upper-half space $\mathbb{R}_{+}^{N}$ and prove a divergence-free atomic decomposition of the space, for this we need to use the even and odd functions. Then we study the Hardy space $\mathcal{H}_{z, d}^{1}\left(\Omega, \wedge^{k}\right)$ when $\Omega$ is $\mathbb{R}_{+}^{N}$ or a special Lipschitz domain. We give its atomic decomposition and characterize its dual space. The key technique is to apply a reflection map defined on the domain $\Omega$. We also establish a "div-curl" type theorem on $\Omega$.

Finally in Chapter VI, we deal with the atomic decomposition and the duality of $\mathcal{H}_{z, d}^{1}\left(\Omega, \wedge^{k}\right)$ when $\Omega$ is a bounded strongly Lipschitz domain.

\section{REFERENCES}

[1] R. Coifman, P.L. Lions, Y. Meyer and S. Semmes, 'Compensated compactness and Hardy spaces', J. Math. Pures Appl. 72 (1993), 247-286.

[2] J.E. Gilbert, J.A. Hogan and J.D. Lakey, 'Atomic decomposition of divergence-free Hardy spaces', in Mathematica Moraviza, Special Volume (Proc. IWAA, 1997), pp. 33-52. http://www.ma. utexas . edu/users/gilbert/papers/Pacificoo.pdf.

[3] P.G. Lemarié-Rieusset, 'Ondelettes vecteurs á divergence nulle', C. R. Acad. Sci. Paris 313 (1991), 213-216.

\footnotetext{
Department of Mathematics

Shantou University

Shantou Guangdong 515063

Peoples Republic of China

e-mail: zjlou@stu.edu.cn
} 